PROCEEDINGS OF THE

AMERICAN MATHEMATICAL SOCIETY

Volume 135, Number 5, May 2007, Pages 1515-1522

S 0002-9939(07)08810-7

Article electronically published on January 9, 2007

\title{
ON THE EXPONENTIAL DECAY OF THE CRITICAL GENERALIZED KORTEWEG-DE VRIES EQUATION WITH LOCALIZED DAMPING
}

\author{
F. LINARES AND A. F. PAZOTO
}

(Communicated by David S. Tartakoff)

\begin{abstract}
This paper is concerned with the asymptotic behavior of solutions of the critical generalized Korteweg-de Vries equation in a bounded interval with a localized damping term. Combining multiplier techniques and compactness arguments it is shown that the problem of exponential decay of the energy is reduced to prove the unique continuation property of weak solutions. A locally uniform stabilization result is derived.
\end{abstract}

\section{INTRODUCTION}

We study the exponential decay of solutions of the critical generalized Kortewegde Vries equation in the domain $(0, L)$ under the presence of a localized damping, that is,

$$
\left\{\begin{array}{l}
u_{t}+u_{x}+u_{x x x}+u^{4} u_{x}+a(x) u=0 \quad \text { in }(0, L) \times(0, \infty), \\
u(0, t)=u(L, t)=0, \quad t \in(0, \infty), \\
u_{x}(L, t)=0, \quad t \in(0, \infty) \\
u(x, 0)=u_{0}(x), \quad x \in(0, L) .
\end{array}\right.
$$

We assume that the real-valued function $a=a(x)$ satisfies the condition

$$
\left\{\begin{array}{l}
a \in L^{\infty}(0, L) \text { and } a(x) \geq a_{0}>0 \text { a.e. in } \Omega, \\
\text { where } \Omega \text { is a nonempty open subset of }(0, L) .
\end{array}\right.
$$

Multiplying (1.1) by $u$ and integrating in $(0, L)$ we get

$$
\frac{d E}{d t}=-\int_{0}^{L} a(x)|u(x, t)|^{2} d x-\frac{1}{2}\left|u_{x}(0, t)\right|^{2}
$$

where

$$
E(t)=\frac{1}{2} \int_{0}^{L}|u(x, t)|^{2} d x
$$

Received by the editors October 24, 2005 and, in revised form, February 24, 2006. 2000 Mathematics Subject Classification. Primary 93D15, 93B05, 35Q53.

Key words and phrases. Exponential decay, stabilization, Korteweg-de Vries equation.

The first author was partially supported by CNPq, Brazil. 
Note that the term $a(x) u$ plays the role of a feedback damping mechanism and, consequently, one can investigate whether the solutions of (1.1) tend to zero as $t \rightarrow \infty$ and the rate at which they decay.

The above equation is a generalization of the well-known Korteweg-de Vries equation, i.e.

$$
u_{t}+u_{x x x}+u u_{x}=0 .
$$

When $a \equiv 0$ the equation in (1.1) considered in the real line is called critical for various reasons. For instance, if $u$ is a solution of the initial value problem associated to the equation in (1.1) with $a \equiv 0$, then $u_{\lambda}(x, t)=\lambda^{1 / 2} u\left(\lambda x, \lambda^{3} t\right)$ is also a solution and $\left\|u_{\lambda}\right\|_{L^{2}}=\|u\|_{L^{2}}$. That is, the mass remains invariant by scaling in the $L^{2}$-norm. Local results for data in $L^{2}$ were established by Kenig, Ponce and Vega [7. On the other hand, in [10, Merle showed that $H^{1}$ solutions may blow-up in finite time. Thus the nonlinearity is critical for the long time behavior of solutions. The generalized Korteweg-de Vries equation has been extensively studied for understanding the interaction between the dispersive term and the nonlinearity in the context of the theory of nonlinear dispersive evolution equations. Here, our purpose is to extend, in some sense, the previous results on the subject obtained for the Korteweg-de Vries equation posed on a finite domain, that is,

$$
u_{t}+u_{x}+u_{x x x}+u u_{x}+a(x) u=0, \quad \text { in }(0, L) \times(0, \infty) .
$$

In this paper, we are concerned with the exponential decay of $E(t)$. More precisely, our purpose is to prove that, for any $R>0$, there exist constants $C=C(R)$ and $\alpha=\alpha(R)$ satisfying

$$
E(t) \leq C(R) E(0) e^{-\alpha(R) t}, \quad \forall t>0,
$$

provided $E(0) \leq R$, which can be stated in the following equivalent form: Find $T>0$ and $C>0$ such that

$$
E(0) \leq C \int_{0}^{T}\left[\int_{0}^{L} a(x) u^{2}(x, t) d x+u_{x}^{2}(0, t)\right] d t
$$

holds for every finite energy solution of (1.1). Indeed, from (1.6) and (1.3) we have that $E(T) \leq \gamma E(0)$ with $0<\gamma<1$, which combined with the semigroup property allow us to derive the exponential decay for $E(t)$. Here, due to technical reasons that will become clear in the proofs, we will consider data $u_{0}$ such that $\left\|u_{0}\right\|_{L^{2}(0, L)}$ is small.

The main tools we use for obtaining (1.6) follow closely the multiplier techniques developed in [12] for the analysis of controllability properties of (1.5) under the boundary conditions given in (1.1). However, when using multipliers, the nonlinearity produces extra terms that we handle by compactness arguments which reduce the problem to showing that the unique solution of (1.1), such that $a(x)=0$ everywhere and $u_{x}(0, t) \in L^{2}(0, L)$ for all time $t$, has to be the trivial one. This problem may be viewed as a unique continuation one since $a u=0$ implies that $u=0$ in $\{a>0\} \times(0, T)$.

The same problem has been intensively investigated in the wave equation context, but there are fewer results for the KdV type equation. The case where the damping term is simultaneously active in a neighborhood of both extremes of the interval $(0, L)$ was addressed in 9 for (1.5) under boundary conditions as in (1.1). By using multiplier techniques the problem was solved in two steps: First, by extending the solution as being zero outside the interval $(0, L)$, one gets a compactly supported 
(in space) solution of the Cauchy problem for the $\mathrm{KdV}$ equation on the whole line. Then, one applies the classical smoothing properties in [6] showing that the solution is smooth. This allows us to apply the unique continuation property results in [17. on smooth solutions to conclude that $u=0$. Later on, performing as in [9], the general case was solved in [11] showing that solutions vanishing on any subinterval are necessarily smooth, which yields enough regularity on $u$ to apply the unique continuation results obtained in 15. More recently, L. Rosier and B.-Y. Zhang in 14 considered the generalized KdV model

$$
u_{t}+u_{x}+u_{x x x}+u^{p} u_{x}+a(x) u=0, \quad \text { in }(0, L) \times(0, \infty)
$$

with boundary conditions as in (1.1) and $p=2,3$. This established existence, uniqueness, and persistence properties of solutions corresponding to the given initial data $u_{0}$, together with continuous dependence on solutions upon the initial data $u_{0}$. Following the methods described above (multiplier techniques, compactness arguments and unique continuation property) the decay of solutions in the energy space was also established. At that point we observe that to obtain the decay of solutions they use a new unique continuation property whose proof is mainly based on a Carleman-type estimate for the Korteweg-de Vries equation established by Rosier in 13 (see also Lemmas 3.1 and 3.2).

In recent years, there has been a great interest in the study of the boundary value problems associated to (1.1) (see for instance, 1, 2], 5], 9, 12, 13]). The difficulty in studying problem (1.1) is introduced by the nonlinearity and the lack of smoothing effects to deal with it. For our purpose it will be enough to consider a weak solution to (1.1) whose existence is guaranteed by Faminskii in 4.

Concerning the exponential decay, we argue as in the previous works, using multipliers that require the application of a unique continuation result. However, this unique continuation result may not be applied directly because of the lack of regularity of the solutions we are dealing with. To overcome this problem, we proceed as in 13 and we first guarantee that solutions are smooth enough (see Lemmas 3.1 and 3.2). Our result is of local nature in the sense that the exponential decay rate is uniform only for initial data in balls $B_{R}(0)$ of $L^{2}(0, L)$ with $0<R<1$.

Before leaving this section we would like to comment on the following issue. For the boundary value problem (1.1), according to the dissipation law (1.3), even when $a \equiv 0$, the energy is dissipated through the extreme $x=0$. In the linear case, as shown in [12] and 9], there are critical lengths $L$ for which the decay is not true. Such lengths $L$ form the set

$$
\mathcal{E}=\left\{\frac{2 \pi}{\sqrt{3}} \sqrt{k^{2}+k l+l^{2}}, k \text { and } l \text { are positive natural numbers }\right\} .
$$

Whether or not solutions of the nonlinear problem decay in this case is an open problem.

The article is organized as follows: In Section 2 we prepare the needed estimates to prove our main result. Section 3 will be devoted to establish the stabilization result.

We would like to thank A. Faminskii for pointing out a minor problem in a previous version of this manuscript and for suggesting the reference [4] which simplified our arguments. 


\section{Preliminary estimates}

We establish a series of estimates that will be useful in the proof of Theorem 3.3. We begin by stating the following existence result due to Faminskii [4.

Theorem 2.1 (See [4], Theorem 1). Let $u_{0} \in L^{2}(0, L)$ and $T>0$ be given. Then, there exists a $T^{*} \in(0, T]$ such that the problem (1.1) admits a unique solution $u \in \mathcal{C}\left(\left[0, T^{*}\right] ; L^{2}(0, L)\right) \cap L^{2}\left(\left(0, T^{*}\right) ; H_{0}^{1}(0, L)\right)$.

In the next proposition we will obtain some additional properties for the solutions of (1.11).

Proposition 2.2. Let $u$ be the solution of problem (1.1) obtained in Theorem 2.1. If $\left\|u_{0}\right\|_{L^{2}(0, L)}<<1$, then

$$
\|u\|_{L^{2}\left(0, T ; H_{0}^{1}(0, L)\right)}^{2} \leq c_{1} \frac{\left\|u_{0}\right\|_{L^{2}(0, L)}^{2}}{\left(1-c_{2}\left\|u_{0}\right\|_{L^{2}(0, L)}^{4}\right)},
$$

where $c_{1}=c_{1}(T, L)$ and $c_{2}$ are positive constants. Furthermore,

$$
u_{t} \in L^{\frac{6}{5}}\left(0, T ; H^{-2}(0, L)\right) .
$$

Proof. The proof will be done in several steps.

First estimate. Multiplying the equation in (1.1) by $u$ we obtain

$$
\frac{1}{2} \frac{d}{d t}\|u(t)\|_{L^{2}(0, L)}^{2}+\frac{1}{2}\left|u_{x}(0, t)\right|^{2}+\int_{0}^{L} a(x)|u(x, t)|^{2} d x=0 .
$$

Consequently, if $T>0$, we deduce that

$$
\|u\|_{L^{\infty}\left(0, T ; L^{2}(0, L)\right)} \leq\left\|u_{0}\right\|_{L^{2}(0, L)} .
$$

Second estimate. Now, we multiply the equation in (1.1) by $x u$ to bound $u$ in $L^{2}\left(0, T ; H_{0}^{1}(0, L)\right)$. Indeed, integrating over $(0, L) \times(0, T)$, we get

$$
\begin{gathered}
\int_{0}^{T} \int_{0}^{L}\left|u_{x}\right|^{2} d x d t+\frac{1}{3} \int_{0}^{L} x|u(x, T)|^{2} d x+\frac{2}{3} \int_{0}^{T} \int_{0}^{L} x a(x)|u|^{2} d x d t \\
=\frac{1}{3} \int_{0}^{T} \int_{0}^{L}|u|^{2} d x d t-\frac{2}{3} \int_{0}^{T} \int_{0}^{L} x u^{5} u_{x} d x d t+\frac{1}{3} \int_{0}^{L} x\left|u_{0}\right|^{2} d x .
\end{gathered}
$$

Then, integrating by parts and using the boundary conditions it follows that

$$
\int_{0}^{T} \int_{0}^{L} x u^{5} u_{x} d x d t=-\frac{1}{6} \int_{0}^{T} \int_{0}^{L}|u|^{6} d x d t .
$$

Thus, replacing (2.10) into (2.9) and using the first estimate we have the following inequality:

$$
\|u\|_{L^{2}\left(0, T ; H_{0}^{1}(0, L)\right)}^{2} \leq \frac{(T+L)}{3}\left\|u_{0}\right\|_{L^{2}(0, L)}^{2}+\frac{1}{9} \int_{0}^{T} \int_{0}^{L}|u(x, t)|^{6} d x d t .
$$

On the other hand, the Gagliardo-Nirenberg inequality and (2.8) imply

$$
\begin{aligned}
\int_{0}^{T} \int_{0}^{L}|u(x, t)|^{6} d x d t & =C \int_{0}^{T}\|u(t)\|_{L^{2}(0, L)}^{4}\left\|u_{x}(t)\right\|_{L^{2}(0, L)}^{2} d t \\
& \leq C\left\|u_{0}\right\|_{L^{2}(0, L)}^{4} \int_{0}^{T}\left\|u_{x}(t)\right\|_{L^{2}(0, L)}^{2} d t
\end{aligned}
$$


for some constant $C$. The above inequality together with (2.11) and the first estimate allow us to conclude that

$$
\left(1-C\left\|u_{0}\right\|_{L^{2}(0, L)}^{4}\right)\|u\|_{L^{2}\left(0, T ; H_{0}^{1}(0, L)\right)}^{2} \leq \frac{(T+L)}{3}\left\|u_{0}\right\|_{L^{2}(0, L)}^{2} .
$$

Third estimate. To obtain a bound for $u_{t}$ we have to pay some attention to the nonlinear term $u^{4} u_{x}=\frac{1}{5} \partial_{x}\left(u^{5}\right)$. First, observe that the argument used in (2.12) gives

$$
\int_{0}^{T} \int_{0}^{L}\left|u^{5}\right|^{6 / 5} d x d t \leq c\left\|u_{0}\right\|^{4} \int_{0}^{T}\left\|u_{x}(t)\right\|_{L^{2}(0, L)}^{2} d t .
$$

Therefore from the first and the second estimates we get

$$
\left\{u^{5}\right\} \text { is bounded in } L^{\frac{6}{5}}((0, T) \times(0, L)) \text {. }
$$

On the other hand, since $L^{\frac{6}{5}}(0, L) \hookrightarrow H^{-1}(0, L)$ we conclude that

$$
\left\{u^{4} u_{x}\right\}=\left\{\frac{1}{5} \partial_{x}\left(u^{5}\right)\right\} \text { is bounded in } L^{\frac{6}{5}}\left(0, T ; H^{-2}(0, L)\right) .
$$

Fourth estimate. Now, we can obtain a bound for $\left\{u_{t}\right\}$. Indeed, since

$$
u_{t}=-u_{x x x}-u_{x}-u^{4} u_{x}-a(x) u
$$

the previous estimates allows to conclude that

$$
\left\{u_{t}\right\} \text { is bounded in } L^{\frac{6}{5}}\left(0, T ; H^{-2}(0, L)\right) \text {. }
$$

This completes the proof of Proposition 2.2

\section{EXPONENTIAL DECAY}

Now we concentrate on the stabilization. The following results will be needed.

Lemma 3.1 (See [14, Lemma 3.6). Let $0<t_{1}<t_{2}<T$ and let $u$ be the solution of (1.1) obtained in Theorem 2.1. Then, there exists a subinterval $\left(t_{1}^{\prime}, t_{2}^{\prime}\right) \subset\left(t_{1}, t_{2}\right)$ such that $u \in L^{\infty}\left(t_{1}^{\prime}, t_{2}^{\prime} ; H^{1}(0, L)\right)$.

Lemma 3.2 (See [14, Lemma 3.5). Let $\Omega$ and $a=a(x)$ be as in (1.2). If $u \in$ $L^{\infty}\left(0, T ; H^{1}(0, L)\right)$ solves (1.1) and $u \equiv 0$ in $\Omega$, then $u \equiv 0$ in $(0, L) \times(0, T)$.

Our main result reads as follows.

Theorem 3.3. Let $u$ be the solution of problem (1.1) given by Theorem 2.1 and let $\Omega$ and $a=a(x)$ be as in (1.2). Then, for any $0<R<1$ and $T>0$, there exist positive constants $c=c(R, T)$ and $\mu=\mu(R)$ such that

$$
E(t) \leq c\left\|u_{0}\right\|_{L^{2}(0, L)}^{2} e^{-\mu t}
$$

holds for all $t>0$ and $u_{0}$ satisfying $\left\|u_{0}\right\|_{L^{2}(0, L)} \leq R$.

Proof. We claim that for any $T>0$, there exists $c=c(T)>0$ such that

$$
\left\|u_{0}\right\|_{L^{2}(0, L)}^{2} \leq c\left[\int_{0}^{T}\left|u_{x}(0, t)\right|^{2} d t+\int_{0}^{T} \int_{0}^{L} a(x) u^{2} d x d t\right]
$$

for every solution of (1.1). This fact, together with the energy dissipation law (1.3) and the semigroup property, suffices to obtain the uniform exponential decay.

To prove (3.13) we first multiply (1.1) by $x u$, integrate over $(0, L) \times(0, T)$ and proceed as in steps (2.9)-(2.11) to get

$$
\|u\|_{L^{2}\left(0, T ; H_{0}^{1}(0, L)\right)}^{2} \leq C(T)
$$


for some $C(T)>0$.

On the other hand, multiplying the equation by $(T-t) u$ and integrating on $(0, L) \times(0, T)$ we obtain the identity

$$
\begin{aligned}
T \int_{0}^{L} u_{0}^{2} d x= & \int_{0}^{T} \int_{0}^{L}|u|^{2} d x d t+\int_{0}^{T}(T-t)\left|u_{x}(0, t)\right|^{2} d t \\
& +2 \int_{0}^{T} \int_{0}^{L}(T-t) a(x)|u|^{2} d x d t .
\end{aligned}
$$

Consequently,

$$
\int_{0}^{L} u_{0}^{2} d x \leq \frac{1}{T} \int_{0}^{T} \int_{0}^{L}|u|^{2} d x d t+\int_{0}^{T}\left|u_{x}(0, t)\right|^{2} d t+2 \int_{0}^{T} \int_{0}^{L} a(x)|u|^{2} d x d t .
$$

Therefore, in order to show (3.13) it suffices to prove that for any $T>0$, there exists a positive constant $C_{1}(T)$ such that

$$
\int_{0}^{T} \int_{0}^{L}|u|^{2} d x d t \leq C_{1}\left\{\int_{0}^{T}\left|u_{x}(0, t)\right|^{2} d t+2 \int_{0}^{T} \int_{0}^{L} a(x)|u|^{2} d x d t\right\} .
$$

Let us argue by contradiction following the so-called "compactness-uniqueness" argument (see for instance [18]). Suppose that (3.17) is not valid. Then, we can find a sequence of functions $\left\{u_{n}\right\} \in L^{\infty}\left(0, T ; L^{2}(0, L)\right) \cap L^{2}\left(0, T ; H_{0}^{1}(0, L)\right)$ that solve (1.1) and such that

$$
\lim _{n \rightarrow \infty} \frac{\left\|u_{n}\right\|_{L^{2}\left(0, T ; L^{2}(0, L)\right)}^{2}}{\int_{0}^{T}\left|u_{n, x}(0, t)\right|^{2} d t+\int_{0}^{T} \int_{0}^{L} a(x) u_{n}^{2} d x d t}=+\infty .
$$

Let $\lambda_{n}=\left\|u_{n}\right\|_{L^{2}\left(0, T ; L^{2}(0, L)\right)}$ and define $w_{n}(x, t)=u_{n}(x, t) / \lambda_{n}$. For each $n \in \mathbb{N}$ the function $w_{n}$ solves

$$
\left\{\begin{array}{l}
w_{n, t}+w_{n, x}+w_{n, x x x}+\lambda_{n}^{4} w_{n}^{4} w_{n, x}+a(x) w_{n}=0 \quad \text { in }(0, L) \times(0, T) \\
w_{n}(0, t)=w_{n}(L, t)=0, \quad t \in(0, T) \\
w_{n, x}(L, t)=0, \quad t \in(0, T) \\
w_{n}(x, 0)=w_{0, n}=u_{n}(x, 0) / \lambda_{n}, \quad x \in(0, L)
\end{array}\right.
$$

Moreover,

$$
\left\|w_{n}\right\|_{L^{2}\left(0, T ; L^{2}(0, L)\right)}=1
$$

and

$$
\int_{0}^{T} \int_{0}^{L}\left|w_{n, x}(0, t)\right|^{2} d x d t+\int_{0}^{T} \int_{0}^{L} a(x) w_{n}^{2} d x d t \longrightarrow 0
$$

as $n \rightarrow \infty$.

Using (3.16) it follows that $w_{n}(\cdot, 0)$ is bounded in $L^{2}(0, L)$. Then, by (3.14) it follows that

$$
\left\|w_{n}\right\|_{L^{2}\left(0, T ; H_{0}^{1}(0, L)\right)} \leq C, \forall n \in \mathbb{N},
$$

for some constant $C>0$. Also, since

$$
w_{n, t}=-w_{n, x}-w_{n, x x x}-\lambda_{n}^{4} w_{n}^{4} w_{n, x}-a(x) w_{n} \text { in } \mathcal{D}^{\prime}\left(0, T ; H^{-2}(0, L)\right)
$$

performing as in the previous section, the above estimates guarantee that

$$
\left\{w_{n, t}\right\} \text { is bounded in } L^{\frac{6}{5}}\left(0, T ; H^{-2}(0, L)\right) \text {. }
$$


At this point, we claim that the following result holds:

There exists $s>0$ such that $\left\{w_{n}\right\}$ is bounded in $L^{4}\left(0, T ; H^{s}(0, L)\right)$, the embedding $H^{s}(0, L) \hookrightarrow L^{4}(0, L)$ being compact.

In fact, since $\left\{w_{n}\right\}$ is bounded in $L^{2}\left(0, T ; H_{0}^{1}(0, L)\right) \cap L^{\infty}\left(0, T ; L^{2}(0, L)\right)$, by interpolation we can deduce that $\left\{w_{n}\right\}$ is bounded in

$$
\left[L^{q}\left(0, T ; L^{2}(0, L)\right), L^{2}\left(0, T ; H_{0}^{1}(0, L)\right)\right]_{\theta}=L^{p}\left(0, T ;\left[L^{2}(0, L), H_{0}^{1}(0, L)\right]_{\theta}\right),
$$

where $\frac{1}{p}=\frac{1-\theta}{q}+\frac{\theta}{2}$ and $0<\theta<1$. Thus, choosing $q=\infty, \theta=1 / 2$, so that $p=4$, the claim holds with $s=1 / 2$, i.e.,

$$
\left[L^{2}(0, L), H_{0}^{1}(0, L)\right]_{\frac{1}{2}}=H^{\frac{1}{2}}(0, L) .
$$

Furthermore, the embedding $H^{\frac{1}{2}}(0, L) \hookrightarrow L^{4}(0, L)$ is compact.

Due to the statement above, (3.22) and classical compactness results ([16, Corollary 4) we can extract a subsequence of $\left\{w_{n}\right\}$, that we also denote by $\left\{w_{n}\right\}$, such that

$$
w_{n} \rightarrow w \text { strongly in } L^{4}\left(0, T ; L^{4}(0, L)\right)
$$

and by (3.19),

$$
\|w\|_{L^{2}\left(0, T ; L^{2}(0, L)\right)}=1
$$

Also,

$$
\begin{aligned}
0 & =\liminf _{n \rightarrow \infty}\left\{\int_{0}^{T}\left|w_{n, x}\right|^{2} d t+\int_{0}^{T} \int_{0}^{L} a(x) w_{n}^{2} d x d t\right\} \\
& \geq \int_{0}^{T}\left|w_{x}(0, t)\right|^{2} d t+\int_{0}^{T} \int_{0}^{L} a(x) w^{2} d x d t .
\end{aligned}
$$

We now distinguish two situations:

(a) There exists a subsequence of $\left\{\lambda_{n}\right\}$ also denoted by $\left\{\lambda_{n}\right\}$ such that

$$
\lambda_{n} \longrightarrow 0 \text {. }
$$

In this case, the limit $w$ satisfies the linear problem

$$
\left\{\begin{array}{l}
w_{t}+w_{x}+w_{x x x}+a(x) w=0 \quad \text { in }(0, L) \times(0, T), \\
w(0, t)=w(L, t)=0, \quad t \in(0, T) \\
w_{x}(L, t)=0, \quad t \in(0, T) \\
w \equiv 0, \quad \text { in } \Omega \times(0, T)
\end{array}\right.
$$

Then, by Holmgren's Uniqueness Theorem, $w \equiv 0$ in $(0, L) \times(0, T)$, and this contradicts (3.24).

(b) There exists a subsequence of $\left\{\lambda_{n}\right\}$ also denoted by $\left\{\lambda_{n}\right\}$ and $\lambda>0$ such that

$$
\lambda_{n} \longrightarrow \lambda \text {. }
$$

In this case, the limit function $w$ solves system (3.18), and so we apply the Unique Continuation Property (UCP) proved in [14] for the subset $\Omega$ obtaining that $w \equiv 0$ in $(0, L) \times(0, T)$, and again this is a contradiction. Indeed, let $t_{1} \in(0, T)$ and let $t_{2} \in\left(t_{1}, T\right)$. According to Lemma 3.1, $w \in L^{\infty}\left(t_{1}^{\prime}, t_{2}^{\prime} ; H^{1}(0, L)\right)$ for some interval $\left(t_{1}^{\prime}, t_{2}^{\prime}\right) \subset\left(t_{1}, t_{2}\right)$. Then, it follows from Lemma 3.2 that $w \equiv 0$ on $\left(t_{1}^{\prime}, t_{2}^{\prime}\right) \times(0, L)$. As $t_{2}$ is arbitrarily close to $t_{1}$, we obtain by continuity of $w$ in $H^{-1}$ that $w(., t)=0$. Thus, $w \equiv 0$. 
In summary, we see that in each of the possible situations (a) and (b) we get a contradiction. Then, necessarily, (3.17) holds. This completes the proof of Theorem 3.3 .

\section{REFERENCES}

[1] J.L. Bona, S.M. Sun and B.-Y. Zhang, A non-homogeneous boundary-value problem for the Korteweg-de Vries Equation posed on a finite domain, Comm. PDE (2003), 1391-1436. MR1998942 (2004h:35195)

[2] T. Colin and J. M. Ghidaglia, An initial-boundary value problem for the Korteweg-de Vries equation posed on a finite interval, Adv. Differential Equations 6 (2001), no. 12, 1463-1492. MR.1858429 (2002i:35160)

[3] J. Coron and E. Crepéau, Exact boundary controllability of a nonlinear KdV equation with critical lengths, J. Eur. Math. Soc. (JEMS) 6 (3)(2004), 367-398. MR2060480 (2005b:93016)

[4] A. V. Faminskii, On an initial boundary value problem in a bounded domain for the Generalized Korteweg-de Vries Equation, Funct. Diff. Eq. 8 (2001) 1-2, 183-194. MR1949998 (2003m:35203)

[5] J. Holmer, The initial-boundary value problem for the Korteweg-de Vries equation, preprint.

[6] T. Kato, On the Cauchy problem for the (generalized) Korteweg-de Vries equation, Advances in Mathematics Supplementary Studies, Studies in Applied Math. 8 (1983) 93-128. MR0759907 (86f:35160)

[7] C. E. Kenig, G. Ponce and L. Vega, Well-posedness and scattering results for the generalized Korteweg-de Vries equation via the contraction principle, Comm. Pure Appl. Math., 46 (1993), 527-620. MR1211741 (94h:35229)

[8] J.L. Lions, Quelques méthodes de résolution des problèmes aux limites non linéaires, Dunod, Paris (1969). MR0259693 (41:4326)

[9] G.P. Menzala, C.F. Vasconcellos and E. Zuazua, Stabilization of the Korteweg-de Vries equation with localized damping, Quarterly of Appl. Math., 60 (1) (2002), 111-129. MR 1878262 $(2002 \mathrm{j}: 35273)$

[10] F. Merle, Existence of blow-up solutions in the energy space for the critical generalized KdV equation, J. Amer. Math. Soc. 14 (2001), 555-578. MR1824989 (2002f:35193)

[11] A. F. Pazoto, Unique continuation and decay for the Korteweg-de Vries equation with localized damping, ESAIM Control Optimization and Calculus of Variations 11 (3) (2005), 473-486. MR2148854(2006b:35292)

[12] L. Rosier, Exact boundary controllability for the Korteweg-de Vries equation on a bonded domain, ESAIM Control Optimization and Calculus of Variations 2 (1997), 33-55. MR.1440078 (98d:93016)

[13] L. Rosier, Control of the surface of a fluid by a wavemaker, ESAIM Control Optimization and Calculus of Variations 10 (2004), 346-380. MR2084328 (2005h:93091)

[14] L. Rosier, B.-Y. Zhang, Global stabilization of the generalized Korteweg-de Vries equation posed on a finite domain, preprint 2005.

[15] J.C. Saut and B. Scheurer, Unique continuation for some evolution equations, J. Diff. Equations 66 (1987), 118-139. MR0871574 (88a:35115)

[16] J. Simon, Compact sets in the space $L^{p}(0, T ; B)$, Annali di Matematica Pura ed Appicata CXLVI (IV) (1987), 65-96. MR0916688 (89c:46055)

[17] B.Y. Zhang, Unique continuation for the Korteweg-de Vries equation, SIAM J. Math. Anal. 23 (1992), 55-71. MR:1145162 (92k:35252)

[18] E. Zuazua, Exponential decay for the semilinear wave equation with locally distributed damping, Comm. Partial Diff. Eq. 15 (2) (1990), 205-235. MR1032629 (91b:35076)

IMPA, Estrada Dona Castorina 110, Rio de Janeiro, 22460-320, Brazil

E-mail address: linares@impa.br

Institute of Mathematics, Federal University of Rio de Janeiro, UfRJ, P.O. Box 68530, CEP 21945-970, Rio de JANeiro, RJ, Brasil

E-mail address: ademir@acd.ufrj.br 